\title{
Tanshinone IIA inhibits the growth, attenuates the stemness and induces the apoptosis of human glioma stem cells
}

\author{
LIUQI YANG, HUIJIE GUO, LIHUA DONG, LI WANG, CHUNLAN LIU and XIUJIE WANG \\ Laboratory of Experimental Oncology, State Key Laboratory of Biotherapy, West China Hospital, \\ West China Medical School, Sichuan University, Chengdu, Sichuan 610041, P.R. China
}

Received March 9, 2014; Accepted May 30, 2014

DOI: 10.3892/or.2014.3293

\begin{abstract}
Glioma stem cells (GSCs) are believed to contribute to glioblastoma multiforme (GBM) propagation and treatment resistance. Tanshinone IIA possesses anticancer and antiinflammatory activities. This study aimed to determine the inhibitory effect of tanshinone IIA on human GSCs in vitro and in vivo and to explore the underlying mechanisms. In the present study, human GBM neurospheres (GBMS) were isolated from adherent GBM cells in serum-free medium, and the cells from the GBMS displayed characteristics of GSCs. Results from the MTT, neurosphere formation and in vivo inhibition assays revealed that tanshinone IIA had a significant inhibitory effect on human GSCs in vitro and in vivo. Furthermore, tanshinone IIA increased the expression of differentiation and neural lineage markers including GFAP and $\beta$-tubulin, decreased expression of GSC markers including CD133 and nestin, and induced GSC apoptosis in vitro and in vivo in a dose-dependent manner. Inflammatory cytokines and signaling pathways are believed to play key roles in maintaining the stem-like properties in human glioma cells. In the present study, inflammatory cytokine interleukin 6 (IL6) and its downstream activated signal transducer and activator of transcription 3 [phospho-STAT3(tyrosine705) and phospho-STAT3(serine727)] were downregulated after tanshinone IIA treatment in vitro and in vivo. This result indicated that disturbance of the IL6/STAT3 signaling axis by tanshinone IIA is closely related to the growth inhibition of GSCs. Taken together, our results indicate that tanshinone IIA has the potential to target and kill GSCs through suppression of proliferation, attenuation of stemness and induction of apoptosis. Its mechanism of activity may be associated with attenuation of the IL6/STAT3 signaling pathway.
\end{abstract}

Correspondence to: Professor Xiujie Wang, Laboratory of Experimental Oncology, State Key Laboratory of Biotherapy, West China Hospital, West China Clinical Medical School, Sichuan University, Chengdu, Sichuan 610041, P.R. China

E-mail: xiujiewang@scu.edu.cn

Key words: glioma stem cells, serum-free medium, tanshinone IIA, IL6/STAT3 signaling pathway, growth inhibition

\section{Introduction}

Glioblastoma multiforme (GBM, grade IV astrocytoma) is considered to be the most highly malignant brain tumor (1). GMB is highly aggressive and currently incurable. While many patients with low-grade gliomas survive for many years, the median survival time of GBM patients is only 12 months (2). The poor prognosis is mainly attributed to the highly diffusive growth pattern of GBM cells into surrounding brain tissue, thereby preventing complete surgical removal (3). There has been a negligible decrease in the mortality rate of GBM patients over the past few decades, indicating that GBM is a complex disease and not easy to cure. Therefore, the development of more effective therapies for GBM must cope with recurrence through elimination of residual single cells or microscopic cell clusters.

Cancer stem cells (CSCs) are a subpopulation of tumor cells with the ability to undergo self-renewal and propagate the tumor population (4). Increasing evidence suggests that CSCs also exist in the GBM population and this CSC subpopulation is referred to as glioma stem cells (GSCs) (5-9). GSCs possess the abilities for self-renewal and multiple differentiation, but also have greater tumorigenic potential than matched non-stem tumor cells when xenotransplanted into immunocompromised rats (7). In addition, GSCs are resistant to chemotherapy and radiotherapy $(10,11)$. Conventional cancer treatments often fail to eliminate GSCs completely, leading to tumor repopulation and relapse $(12,13)$. Thus, targeting GSCs is considered a novel therapeutic avenue to more effectively eradicate malignant tumors and reduce the risk of relapse.

Tanshinone IIA is a lipophilic diterpene isolated from Salvia miltiorrhiza (Danshen), a widely used Chinese herbal medicine (14). A number of studies have shown that tanshinone IIA possesses anticancer $(15,16)$, anti-inflammatory $(17,18)$ and antioxidative (19) activities in vitro and in vivo through the inhibition of special signaling pathways $(20,21)$, suggesting it may be an effective chemotherapeutic agent for malignant tumor therapy.

Previous studies have shown that CSCs are maintained by inflammatory signaling pathways and the microenvironment $(22,23)$ in which inflammatory cytokine interleukin 6 (IL6) (22) plays an important role. Tanshinone IIA was found to exhibit a potent inhibitory effect on the expression of inflammatory cytokines $(21,24)$. Therefore, the anti-inflammatory activity of tanshinone IIA 
may be a potential therapeutic strategy for tumor inhibition through the downregulation or attenuation of the expression of inflammatory cytokines.

In the present study, we investigated the effect of serumfree medium (SFM) on GSC formation and examined the effects of tanshinone IIA on human GSCs in vitro and in vivo, and aimed to elucidate the potential molecular mechanisms of its antitumor effect.

\section{Materials and methods}

Cell culture and clone formation. Human WJ1 (25) cells were cultured in DMEM/F12 supplemented with 10\% FBS (SCM), penicillin $(100 \mathrm{U} / \mathrm{ml})$ and streptomycin $(100 \mu \mathrm{g} / \mathrm{ml})$ in a humidified atmosphere of $50 \mu \mathrm{g} / \mathrm{ml} \mathrm{CO}_{2}$ at $37^{\circ} \mathrm{C}$ until formation of an adherent monolayer of GBM cells. Then cells from the serum-grown cultures were dissociated and cultured with serum-free DMEM/F12 medium supplemented with $20 \mathrm{ng} / \mathrm{ml}$ EGF, $20 \mathrm{ng} / \mathrm{ml} \mathrm{bFGF}$, and 1X B27 (Invitrogen, Carlsbad, CA, USA), until primary neurosphere formation (26). Neurospheres were photographed using an inverted microscope after neurosphere formation.

$R T-P C R$. Total RNA was harvested from cells using TRIzol reagent (CWBIO). PCR was performed on cDNA generated by HIFI-MMLV reverse transcriptase (CWBIO) in a total reaction volume of $20 \mu \mathrm{l}$ according to the manufacturer's instructions. The sequences of forward and reverse oligonucleotide primers, specific to the chosen candidates and housekeeping genes, were used as follows: CD133 forward, 5'-CGACTGAGACCCAACATC-3' and reverse, 5'-CCCTTTT GATACCTGCTACGAC-3'; GFAP forward, 5'-CGATCAACT CACCGCCAACA-3' and reverse, 5'-GTGGCTTCATCTGCT TCCTGTC-3'; GAPDH forward, 5'-ACCACAGTCCATGCCA TCAC-3' and reverse, 5'-TCCACCACCCTGTTGCTGTA-3'. All data were normalized to GAPDH transcript levels.

Western blotting. Western blotting was performed as previously described (3). Rabbit polyclonal antibodies for human CD133 (1:500 dilution; Signalway Antibody, Preland,TX, USA), nestin, GFAP, $\beta$ III-tubulin, IL6, Bax, cleaved caspase-3, Bcl2, signal transducer and activator of transcription 3 (STAT3), phospho-STAT3(tyrosine705) and phospho-STAT3(serine727) (1:500 dilution; Bios Biotechnology, Beijing, China) were used according to the manufacturer's instructions. Antibody recognition was detected with the peroxidase-conjugated goat anti-rabbit IgG (H+L) secondary antibody (Zhongshan Goldenbridge Biotechnology, Beijing, China) using a 1:3,000 dilution. Antibody-bound proteins were detected by the BeyoECL Plus kit (Beyotime Institute of Biotechnology, Shanghai, China) and western blotting system (Universal Hood II, Bio-Rad, USA), and normalized to $\beta$-actin and quantified using the ChemiDoc ${ }^{\mathrm{TM}}$ XRS (Bio-Rad).

Tumorigenicity assay. To evaluate the tumorigenicity of the GBMS cells, $1 \times 10^{4}, 1 \times 10^{5}, 1 \times 10^{6}$ of GBM cells and $1 \times 10^{2}, 1 \times 10^{3}$, $1 \times 10^{4}$ of GBMS cells were suspended in $50 \mu 1$ Matrigel (BD Biosciences) and $50 \mu \mathrm{l}$ PBS, and then cells were implanted bilaterally into the flank of BALB/c nu/nu mice. Animals were maintained under standard conditions according to the guidelines of the Institutional Animal Care and Use Committee of Sichuan University. Tumor incidence was recorded 3 times weekly after the injection. Mice were maintained on a standard rodent chow and had free access to water. Tumors of the euthanized mice were collected, and their size and weight were measured.

Inhibition of colony formation. Single-cell suspensions of GBMS were seeded in a 96-well plate at a density of 100 cells per well. Cells were treated with tanshinone IIA at concentrations of $0.25,0.5$ and $1.0 \mu \mathrm{g} / \mathrm{ml}$ respectively. Each concentration was repeated in 6 wells. After a 7-day incubation at $37^{\circ} \mathrm{C}$ in a humidified $5 \% \mathrm{CO}_{2}$ atmosphere, the number of neurospheres in each well was quantified under a microscope. Three independent experiments were performed.

MTT assay. The 3-[4,5-dimethylthiazol-2-yl]-2, 5 diphenyltetrazolium bromide (MTT) assay was performed as previously described (27). GBMS, GBM and LO2 (normal control) cells ( $2 \times 10^{3} /$ well) were seeded in $100 \mu \mathrm{l}$ of medium/well in 96 -well plates. After a 24-h incubation, tanshinone IIA was added at various concentrations $(0.125,0.25,0.5,1.0$ and $2.0 \mu \mathrm{g} / \mathrm{ml})$; 5 wells were included for each concentration. The effects of tanshinone IIA on the viability of GBMS, GBM and normal cells was expressed as the \% cytoviability, using the following formula: $\%$ cytoviability = A490 of the treated cells/A490 of the control cells (without tanshinone IIA) x $100 \%$ (28). Three independent experiments were performed.

Annexin V-FITC assay. In order to determine apoptosis in GBMS cells, the Annexin V-FITC apoptosis detection kit (KeyGen Biotech, Nanjing, China) was used according to the manufacturer's instructions. The extent of apoptosis in the samples was measured by flow cytometry using BD FACSCalibur flow cytometer and CellQuest software. Three independent experiments were performed.

In vivo inhibition assay. The flank xenografts were established by subcutaneous injections of $10^{4}$ GSCs per flank ( 5 mice per group). Animals were maintained under standard conditions according to the guidelines of the Institutional Animal Care and Use Committee of Sichuan University. After one week of inoculation, the tumor-bearing mice were randomized into four groups, each having 5 mice. The mice of the three treatment groups were injected with 10,20 and $40 \mathrm{mg} / \mathrm{kg}$ of tanshinone IIA i.p. 3 times a week for 8 weeks, respectively, and the control mice were injected with an equal volume of blank dissolvant. In the experimental process, mice were weighed, and tumor volumes were assessed each week. At the end of the experiment, the mice were sacrificed by carbon dioxide asphyxiation and tumors were harvested, weighed and examined. The tumor inhibitory rate was calculated using the following formula: Tumor inhibitory rate $(\%)=$ (mean tumor weight of the control mice - mean tumor weight of the treated mice)/average tumor weight of the control mice $\mathrm{x} 100 \%$.

Statistical analysis. Results are expressed as means \pm standard deviation. To evaluate the significant differences between two groups, the means were compared using the Student's t-test. Multiple group comparisons were performed using one-way 

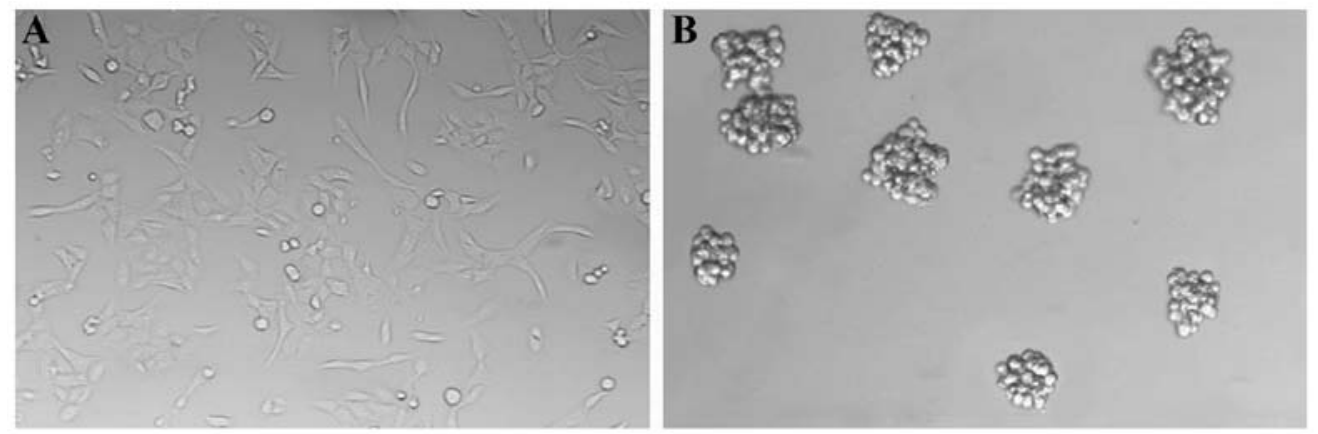

Figure 1. Morphology of GBM and GBMS cells. (A) GBM cells cultured in SCM grew as an adherent monolayer. (B) GBM cells cultured in SFM formed primary neurosphere-like colonies after 10-14 days.
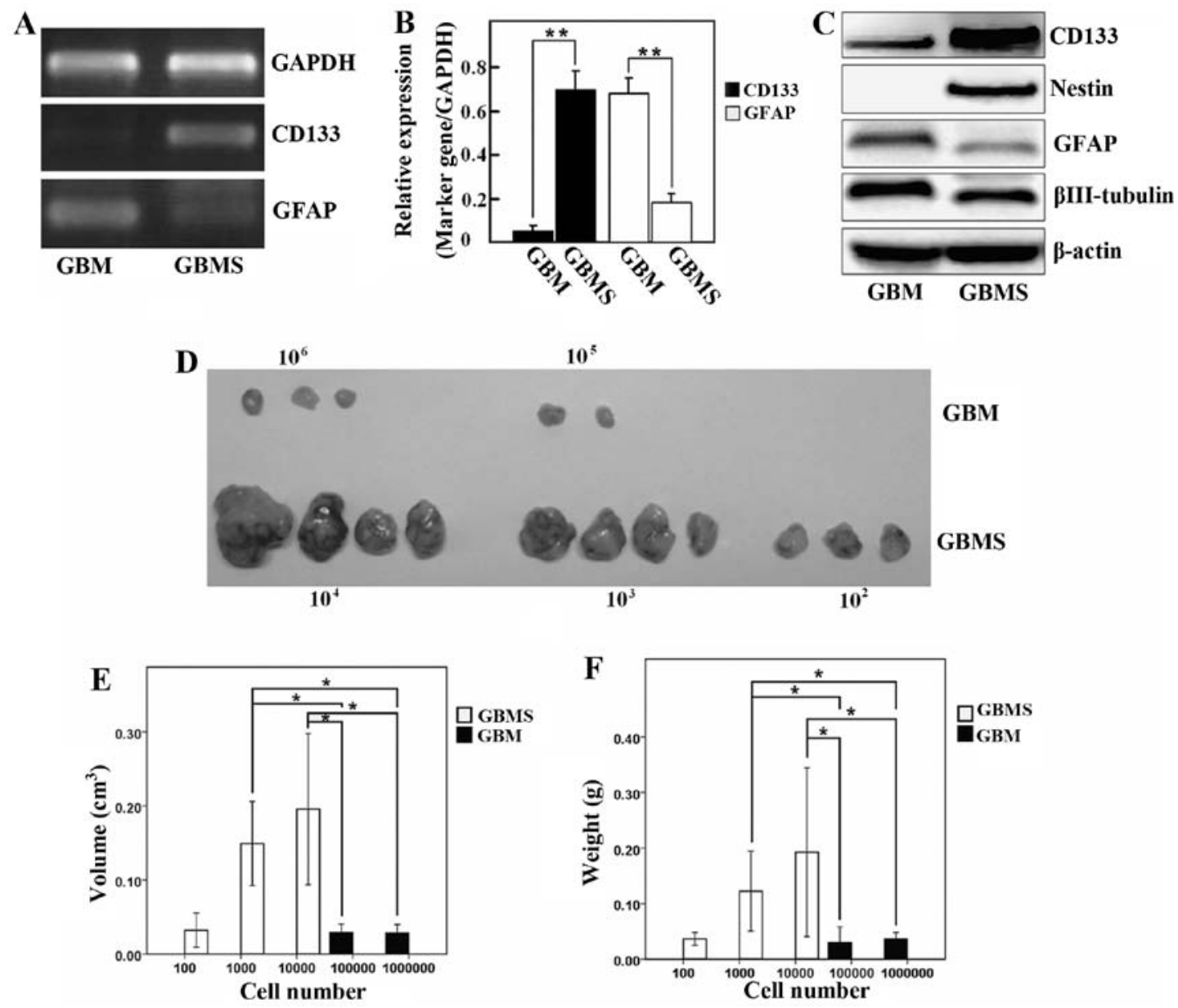

$10^{5}$

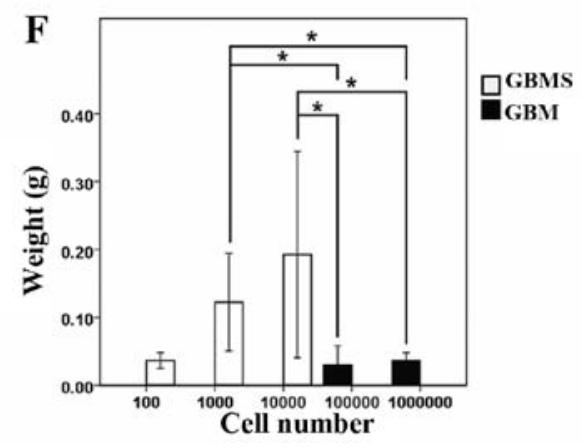

Figure 2. GBMS exhibit characteristics of GSCs. (A) A higher CD133 and a lower GFAP transcript were noted in GBMS when coupled with those in GBM cells with GAPDH included as a reference control. (B) The histogram shows significantly higher CD133 and lower GFAP transcript in GBMS than those in the adherent GBM cells; ${ }^{* *} \mathrm{P}<0.01$. (C) Western blot analysis revealed higher CD133 and nestin, lower GFAP and $\beta$-tubilin III in GBMS than these levels in the adherent GBM cells with $\beta$-actin included as the reference control. (D) Tumor masses from the GBM group (implantation with $1 \times 10^{6}, 1 \times 10^{5}$ and $1 \times 10^{4}$ GBM cells, respectively) and from the GBMS group (implanted with $1 \times 10^{4}, 1 \times 10^{3}$ and $1 \times 10^{2}$ GBMS cells, respectively). The histogram shows that there was a significant difference in (E) mean tumor volume and (F) mean tumor weight between the GBMS and GBM group; ${ }^{*} \mathrm{P}<0.05$.

analysis of variance. The level of statistical significance was defined as $\mathrm{P}<0.05$ for all tests. All statistical analyses were performed using SPSS 16.0 (SPSS, Inc., Chicago, IL, USA)

\section{Results}

GBMS exhibit characteristics of GSCs in vitro. GBM cells exhibited adherent growth in SCM (Fig. 1A). We switched the
GBM cells into culture conditions for GSC proliferation in vitro. Primary neurosphere-like colonies (GBM neurospheres; GBMS) were observed in the SFM after 10-14 days (Fig. 1B). We next digested these neurosphere-like colonies into single-cell suspensions and were seeded at a clonal density in the SFM in a 24-well plate. Subspheres appeared after culturing for 7-10 days. This result indicated that GBMS contained individual stem-like cells with the ability to self-renew and form new spheres. 

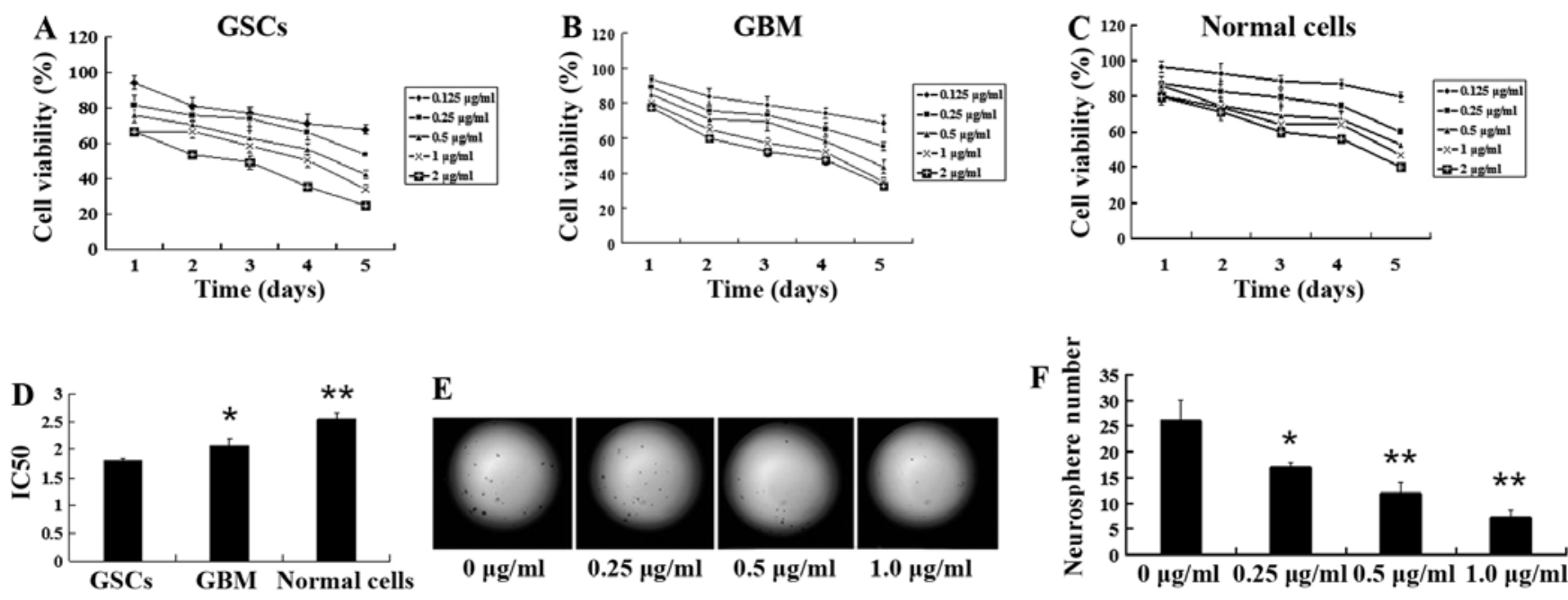

Figure 3. Tanshinone IIA suppresses neurosphere formation and proliferation of GSCs. GBMS, GBM and LO2 (normal control) cells were treated with 0.125, $0.25,0.5,1.0$ and $2.0 \mu \mathrm{g} / \mathrm{ml}$ of tanshinone IIA and incubated for 1, 2, 3, 4 and 5 days, respectively. Cell viability was detected by MTT assay, using the method indicated in Materials and methods. Tanshinone IIA exhibited a dose- and time-dependent inhibitory effect on (A) GBMS, (B) GBM and (C) normal control cells. Tanshinone IIA exhibited a more significant inhibitory effect in the GBMS cells than in the GBM and LO2 cells. (D) The half inhibitory concentration $\left(\mathrm{IC}_{50}\right)$ values for the GSCs, GBM and normal cells after treatment with tanshinone IIA for 3 days are shown $\left(72 \mathrm{~h} ;{ }^{*} \mathrm{P}<0.05\right.$, $\left.{ }^{* *} \mathrm{P}<0.01\right)$. (E) GSCs were plated in 96-well plates at a density of 100 cells/well and treated with the indicated concentrations of tanshinone IIA. Representative examples of neurospheres from GSCs on day 7 are shown. Tanshinone IIA decreased neurosphere number in a dose-dependent manner (magnification x200). (F) The histogram shows that there was a significant decrease in the number of neurospheres after tanshinone IIA treatment in a dose-dependent manner; ${ }^{* *} \mathrm{P}<0.01$. Results are mean values $\pm \mathrm{SD}$ of independent experiments performed in triplicate.

The level of the CD133 transcript was significantly higher in the GBMS when compared with the GBM cells. In contrast, the GFAP transcript was obviously lower in the spheres than that in the GBM cells (Fig. $2 \mathrm{~A}$ and $\mathrm{B}$; $\mathrm{P}<0.05$ ). Western blotting data showed that the protein levels of stemness and differentiation markers of GSCs were in agreement with the immunofluorescence and gene expression (Fig. 2C). These results indicated that the stem-like characteristic of GBMS were enriched when grown in SFM.

GBMS display higher tumorigenicity than GBM cells in vivo. To confirm the different tumor-initiating capabilities between GBMS and GBM cells in vivo, both spheres $\left(1 \times 10^{2}, 1 \times 10^{3}\right.$, $1 \times 10^{4}$ cells) and adherent monolayer cells $\left(1 \times 10^{4}, 1 \times 10^{5}, 1 \times 10^{6}\right.$ cells) were implanted bilaterally into the flank of BALB/c nu/ nu mice for analysis of transplanted tumorigenicity. GBMS cells generated tumors when only $1 \times 10^{2}$ cells were injected into mice. In contrast, at least $1 \times 10^{5} \mathrm{GBM}$ cells were needed to generate tumors, suggesting that GBMS contain more tumor-initiating cells by at least 1,000-fold when compared to this number in the parental cells. A comparative analysis of gross appearance revealed the presence of significant differences regarding the size and mass between the tumors newly generated from the GBM and GBMS cells after implantation for 6 weeks (Fig. 2D-F; P<0.05). This result indicates that the tumorigenicity of GBMS cells was significantly higher than that of the GBM cells.

Tanshinone IIA inhibits GSC (GBMS) proliferation in vitro. To determine whether tanshinone IIA suppresses GSC proliferation, we compared the inhibitory effect of tanshinone IIA on human GSCs, GBM and normal control (LO2) cells and under proper drug concentrations after treatment for 1-5 days, respectively. The inhibition of tanshinone IIA at various concentrations in these cells was determined as the ratio of the number of viable treated cells to the number of viable cells of the untreated controls. Tanshinone IIA exhibited a doseand time-dependent inhibitory effect on GSCs, GBM and normal cells (Fig. 3A-C). The $\mathrm{IC}_{50}$ value was $2.542 \pm 0.34 \mu \mathrm{g} /$ $\mathrm{ml}$ for normal cells, $2.066 \pm 0.135 \mu \mathrm{g} / \mathrm{ml}$ for GBM cells and $1.812 \pm 0.196 \mu \mathrm{g} / \mathrm{ml}$ for GSCs, respectively, after $72 \mathrm{~h}$ of treatment (Fig. 3D; $\mathrm{P}<0.05$ ). Our results showed that, compared with GBM and normal cells, GSCs were more sensitive to tanshinone IIA in vitro, suggesting that tanshinone IIA possesses stronger inhibitory activity against GSCs.

Tanshinone IIA inhibits neurosphere formation. To test the inhibitory effect of tanshinone IIA on neurosphere formation in GSCs, we incubated single-cell suspensions of GBMS with escalating concentrations of tanshinone IIA and evaluated neurosphere formation on day 7 . We found that there was a dosedependent reduction in neurosphere formation after treatment with tanshinone IIA (Fig. 3E). The mean number of neurospheres in the control were $26.0 \pm 4.0$ after seeding 100 cells per well, whereas the mean numbers of neurospheres treated with $0.25,0.5$ and $1.0 \mu \mathrm{g} / \mathrm{ml}$ of tanshinone IIA for $48 \mathrm{~h}$ were $17.0 \pm 1.0(\mathrm{P}<0.05), 12.0 \pm 2.0(\mathrm{P}<0.01)$ and $7.33 \pm 1.15(\mathrm{P}<0.01)$, respectively (Fig. 3F). A significant reduction in the number of neurospheres was observed at concentrations of tanshinone IIA as low as $0.25 \mu \mathrm{g} / \mathrm{ml}$. Following exposure to tanshinone IIA at a dose of $1.0 \mu \mathrm{g} / \mathrm{ml}$, neurosphere formation of GSC decreased sharply.

Tanshinone IIA inhibits the growth of GSC-derived tumors. To investigate the inhibitory activity of tanshinone IIA on human GSCs in vivo, human GSC-bearing mice were injected 

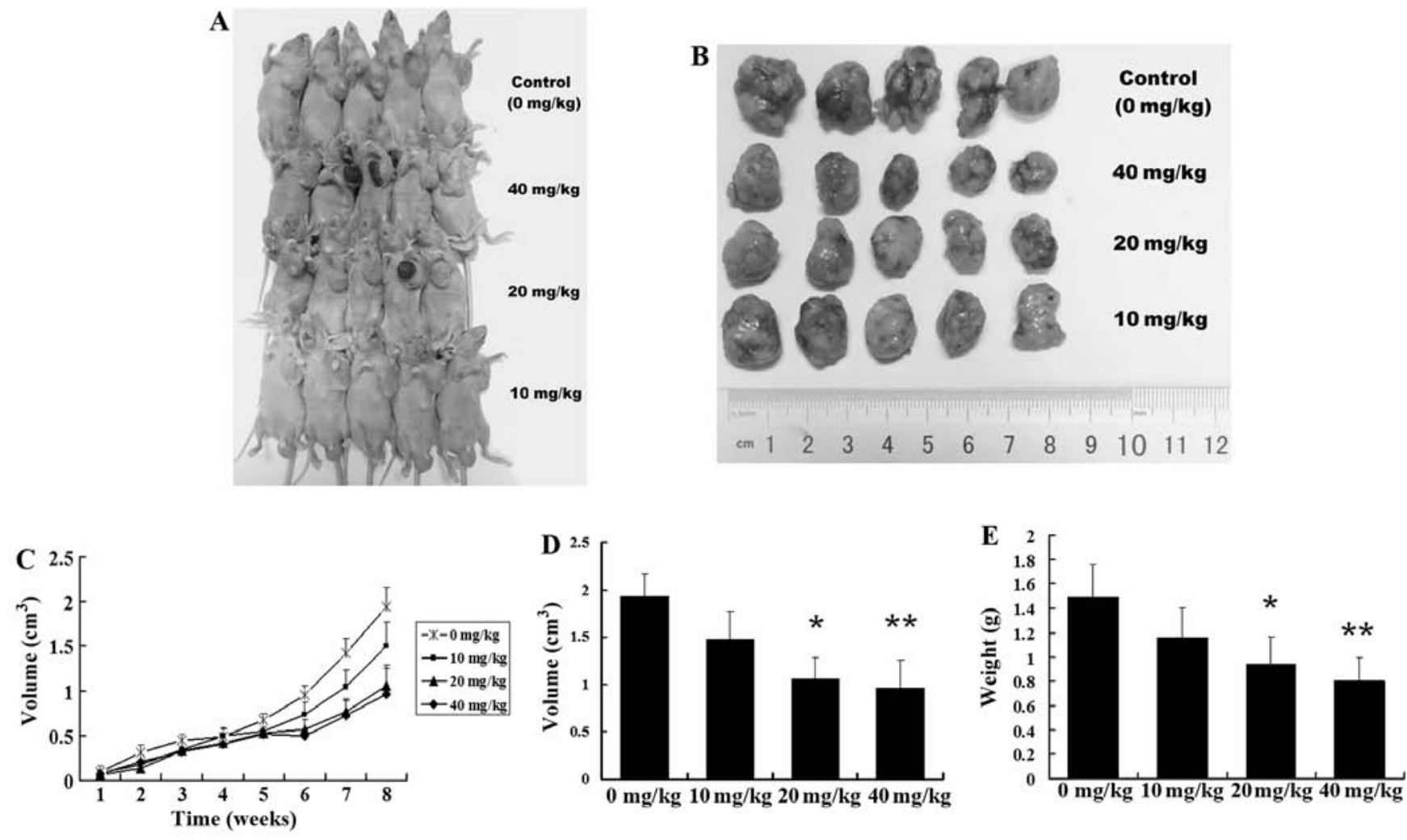

Figure 4. Tanshinone IIA inhibits human brain tumor growth initiated by GSCs in vivo. (A) Tumor-bearing mouse models. (B) Tumor masses from each group. (C) The change in tumor volume in each group after tanshinone IIA treatment. Histograms show that there were significant differences in (D) mean tumor volume and (E) mean tumor weight between the tanshinone IIA-treated group and the untreated control; $\mathrm{P}<0.05 ;{ }^{* *} \mathrm{P}<0.01$.

A

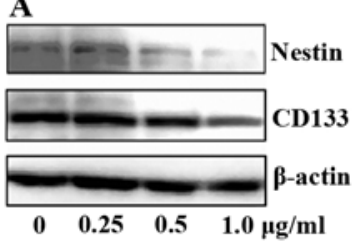

B

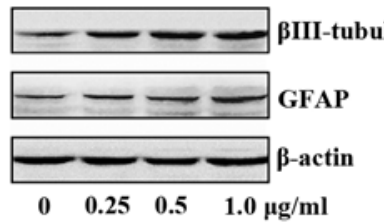

D

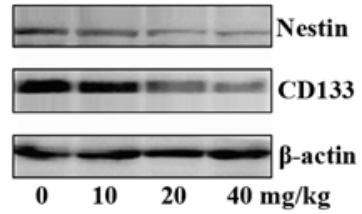

$\mathbf{E}$

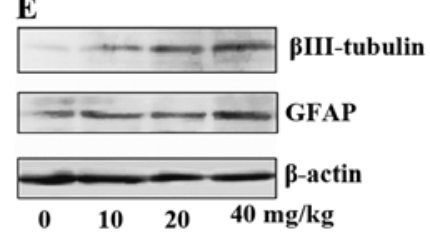

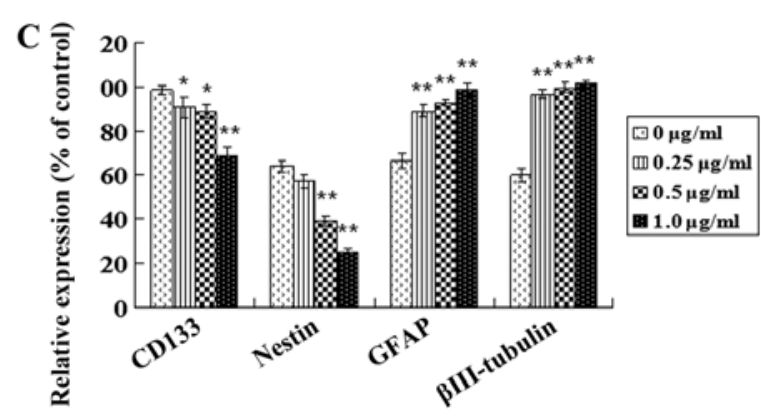

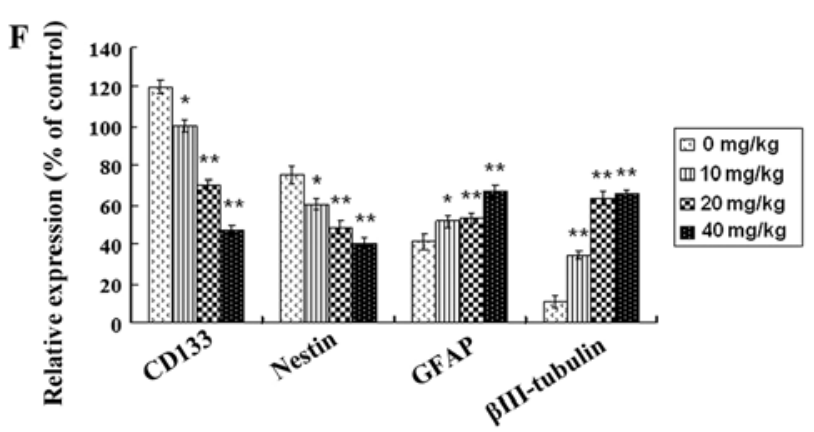

Figure 5. Tanshinone IIA regulates protein expression in connection with GSC stemness and differentiation. Human GSCs were treated with tanshinone IIA $(0.25,0.5$ and $1.0 \mu \mathrm{g} / \mathrm{ml})$ for $48 \mathrm{~h}$, respectively. (A) Protein expression levels of CD133 and nestin were downregulated while (B) GFAP and $\beta$ III-tubulin were upregulated in vitro in a dose-dependent manner. (C) The histogram shows that there was a significant decrease in CD133 and nestin, but an obvious increase in GFAP and $\beta I I I-t u b u l i n$ in vitro in a dose-dependent manner after tanshinone IIA treatment; ${ }^{*} \mathrm{P}<0.05,{ }^{* *} \mathrm{P}<0.01$. Human GSC bearing-mice were injected with 10,20 , and $40 \mathrm{mg} / \mathrm{kg}$ of tanshinone IIA i.p. 3 times a week for 8 weeks. (D) The protein expression levels of CD133 and nestin in tumors were downregulated, and (E) GFAP and $\beta$ III-tubulin were upregulated in a dose-dependent manner. (F) The histogram shows that there was a significant decrease in CD133 and nestin, but a significant increase in GFAP and $\beta$ III-tubulin in vivo in a dose-dependent manner after tanshinone IIA treatment; ${ }^{*} \mathrm{P}<0.05,{ }^{* *} \mathrm{P}<0.01$.

with 10,20 or $40 \mathrm{mg} / \mathrm{kg}$ of tanshinone IIA i.p. 3 times a week for 8 weeks. Tanshinone IIA potently inhibited the growth of GSC-derived xenografts with a reduction in tumor weight and volume (Fig. 4A-C). In contrast, the controls were not significantly inhibited in terms of tumor growth. The mean tumor volume of the control mice was $1.92 \pm 0.40 \mathrm{~cm}^{3}$, and 
A
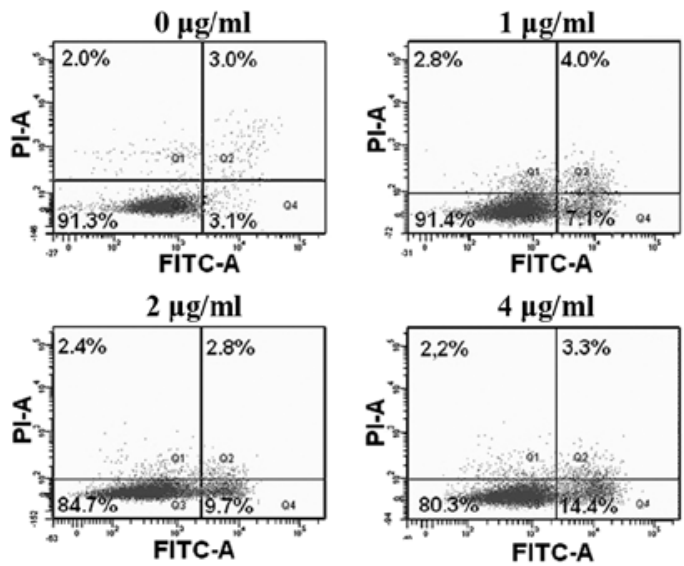

C

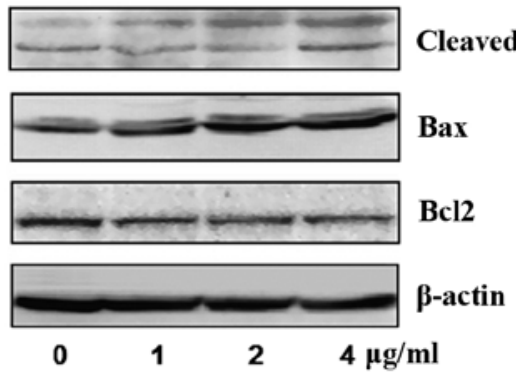

$\mathbf{E}$

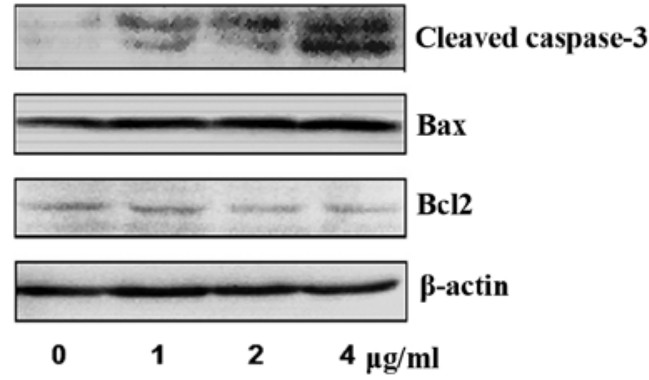

B

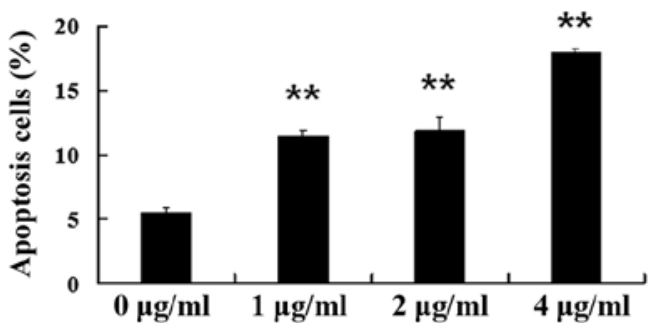

D

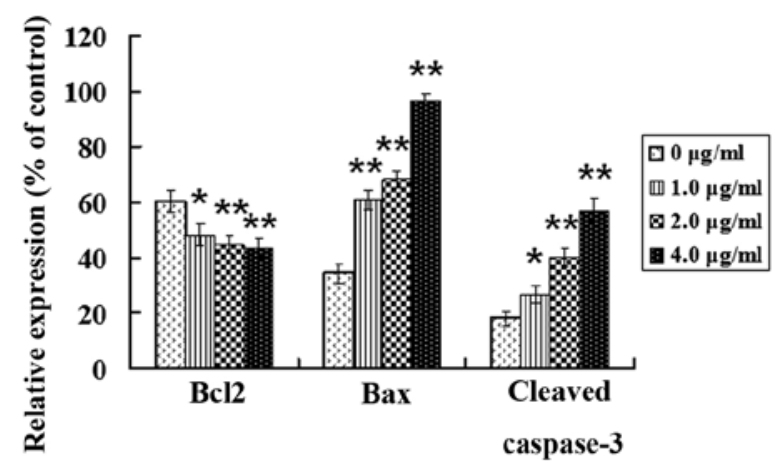

F

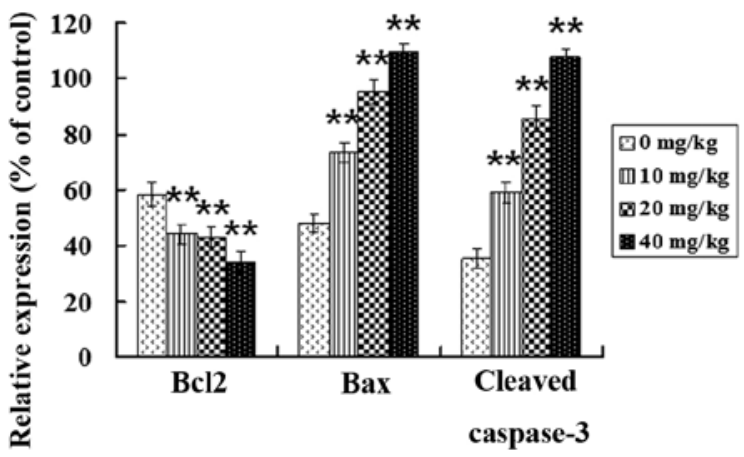

Figure 6. Tanshinone IIA induces GSC apoptosis. (A) Following treatment with tanshinone IIA (1.0, 2.0 and $4.0 \mu \mathrm{g} / \mathrm{ml})$ for $24 \mathrm{~h}$, respectively, apoptosis in GSCs was detected by flow cytometry. (B) Apoptotic rate was calculated. (C) GSCs were treated with tanshinone IIA (1.0, $2.0 \mathrm{and} 4.0 \mu \mathrm{g} / \mathrm{ml})$ for $24 \mathrm{~h}$, respectively. The levels of apoptotic proteins Bax and cleaved caspase-3 were upregulated, while the expression of anti-apoptosis protein Bcl2 was downregulated in a dose-dependent manner. (D) The histogram shows that there was a significant increase in Bax and cleaved caspase-3, and a significant decrease in Bcl2 in vitro in a dose-dependent manner after tanshinone IIA treatment; ${ }^{*} \mathrm{P}<0.05,{ }^{* *} \mathrm{P}<0.01$. (E) The GSC-bearing mice were injected with $10,20 \mathrm{or} 40 \mathrm{mg} / \mathrm{kg}$ of tanshinone IIA for 8 weeks, respectively, and the levels of the apoptotic proteins were examined in tumor tissues. Bax, cleaved caspase-3 were upregulated, while Bcl2 was downregulated in a dose-dependent manner. (F) There was a significant increase in Bax and cleaved caspase-3, and a significant decrease in $\mathrm{Bcl} 2$ in vivo in a dose-dependent manner after tanshinone IIA treatment; ${ }^{*} \mathrm{P}<0.05,{ }^{* *} \mathrm{P}<0.01$.

those of the mice injected with 10,20 , and $40 \mathrm{mg} / \mathrm{kg}$ of tanshinone IIA were $1.34 \pm 0.49,1.02 \pm 0.38(\mathrm{P}<0.05)$ and $0.90 \pm 0.36 \mathrm{~cm}^{3}(\mathrm{P}<0.01)$, respectively (Fig. 4D). The mean tumor weight of the control mice was $1.49 \pm 0.27 \mathrm{~g}$, and those of the mice injected with 10,20 and $40 \mathrm{mg} / \mathrm{kg}$ of tanshinone IIA were $1.16 \pm 0.24,0.94 \pm 0.22(\mathrm{P}<0.05)$ and $0.80 \pm 0.20 \mathrm{~g}(\mathrm{P}<0.01)$, respectively (Fig. 4E). The tumor inhibitory rates were 22.14 , $36.91(\mathrm{P}<0.05)$ and $46.30 \%(\mathrm{P}<0.05)$, respectively. No evidence of toxicity was identified in the treated animals by comparing the body weight increase, histopathological changes in major organs, and blood biochemistry analysis of both the control and the treated group animals (data not shown).
Tanshinone IIA attenuates GSC stemness and induces GSC apoptosis. After treatment with tanshinone IIA, protein expression levels of GSC markers including CD133 and nestin and differentiation and neural lineage markers including GFAP for astrocytes and $\beta$ III-tubulin for neurons in vitro and in vivo were analyzed. CD133 and nestin in GSCs were decreased both in vitro and in vivo in a dose-dependent manner (Fig. 5A and D; Fig. 5C and F; P<0.05). In contrast, the levels of GFAP and $\beta$ III-tubulin were increased in a dosedependent manner (Fig. 5B and E; Fig. 5C and F; P<0.05). Furthermore, tanshinone IIA treatment resulted in th apoptosis of GSCs. As shown in Fig. 6A, the number of apoptotic 

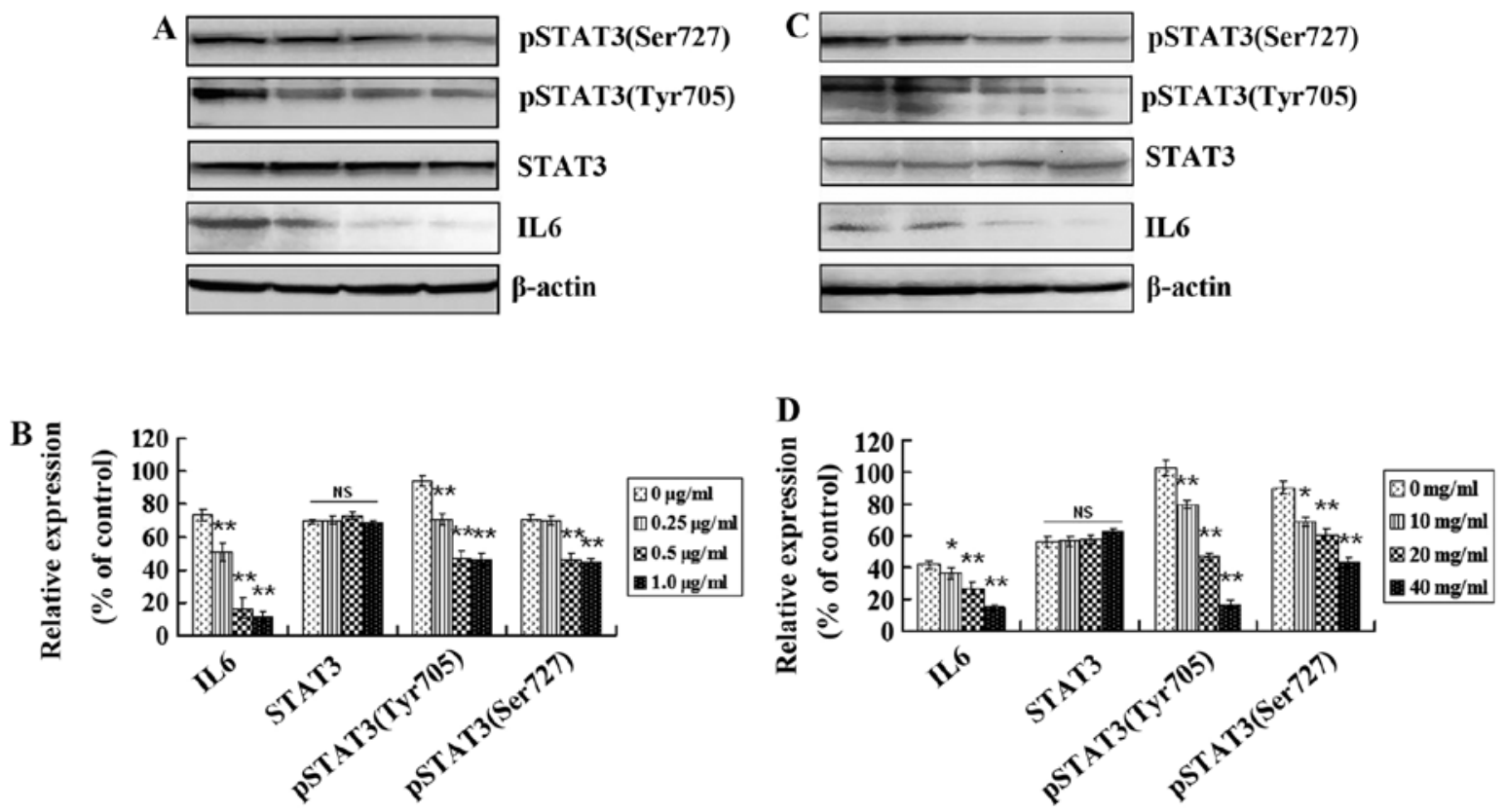

Figure 7. Tanshinone IIA regulates protein expression mediating inflammatory signaling pathways in GSCs. (A) Western blot analysis of IL6, STAT3, phospho-STAT3(tyrosine 705) and phospho-STAT3(serine727) proteins before and after tanshinone IIA (0.25, 0.5 and $1.0 \mu \mathrm{g} / \mathrm{ml})$ treatment for $48 \mathrm{~h}$. (B) The histogram shows that there was no change in STAT3 but a significant decrease in IL6, phospho-STAT3(tyrosine705) and phospho-STAT3(serine727) in vitro in a dose-dependent manner after tanshinone IIA treatment; ${ }^{*} \mathrm{P}<0.05,{ }^{* *} \mathrm{P}<0.01$. (C) Western blot analysis of IL6, STAT3, phospho-STAT3(tyrosine705) and phospho-STAT3(serine727) proteins before and after tanshinone IIA treatment (10, 20, and $40 \mathrm{mg} / \mathrm{kg}$ ) in vivo. (D) There was no change in STAT3 but a significant decrease in IL6, phospho-STAT3(tyrosine705) and phospho-STAT3(serine727) in vivo in a dose-dependent manner after tanshinone IIA treatment; ${ }^{*} \mathrm{P}<0.05,{ }^{* *} \mathrm{P}<0.01$.

cells was significantly increased following treatment with tanshinone IIA. Tanshinone IIA exposure resulted in a more than 3 -fold increase in Annexin V-positive cells (Fig. 6B), which was highly significant $(\mathrm{P}<0.01)$. Moreover, expression levels of apoptosis-related proteins were examined in vitro and in vivo after treatment with tanshinone IIA. Bax and cleaved caspase-3 (apoptotic proteins) were increased and anti-apoptotic protein $\mathrm{Bcl}-2$ was decreased in a dose-dependent manner after treatment with $1.0,2.0$ or $4.0 \mu \mathrm{g} / \mathrm{ml}$ of tanshinone IIA for $24 \mathrm{~h}$ in vitro (Fig. 6C and D; $\mathrm{P}<0.05$ ). Apoptosis-related proteins in tumors from human GSC bearing-mice were examined after treatment with 10,20 and $40 \mathrm{mg} / \mathrm{kg}$ of tanshinone IIA for 8 weeks. Bax and cleaved caspase-3 were increased, and $\mathrm{Bcl}-2$ was decreased in a dose-dependent manner (Fig. 6E and F; $\mathrm{P}<0.05$ ), which was in consistent with the in vitro results. These results indicate that tanshinone IIA has the potential not only to attenuate GSC stemness, but also to induce GSC apoptosis in vitro and in vivo.

Tanshinone IIA regulates protein expression mediating inflammatory signaling pathways in GSCs. Inflammatory cytokines play important roles in the maintenance and progression of CSCs. To understand the molecular mechanisms underlying the inhibitory effect of tanshinone IIA on GSCs, we examined protein expression profiles mediating inflammatory signaling pathways. Inflammatory cytokine IL6, STAT3 and the active form of STAT3 [(phospho-STAT3(tyrosine705) and phosphoSTAT3(serine727)] in GSCs in vitro and in vivo were analyzed using western blotting.Expression levels of IL6, phosphoSTAT3(tyrosine705) and phospho-STAT3(serine727) were higher in the GSCs from cells and tumor tissues (Fig. 7A and C, respectively). IL6, phospho-STAT3(tyrosine705) and phosphoSTAT3(serine727) were decreased in vitro after treatment with $0.25,0.5$ and $1.0 \mu \mathrm{g} / \mathrm{ml}$ of tanshinone IIA for $48 \mathrm{~h}$ and in vivo after treatment with 10,20 and $40 \mathrm{mg} / \mathrm{kg}$ of tanshinone IIA for 8 weeks (Fig. 7B and D; P<0.05). However, no significant expression change in STAT3 was observed after treatment with different concentrations of tanshinone IIA (Fig. 7B and D; $P>0.05$ ). These results suggest that IL6 and downstream activated STAT3 play roles in GSC growth, and tanshinone IIA has the potential to block inflammatory signaling pathways in GSCs by downregulating pathway-related protein expression.

\section{Discussion}

GBM is an extremely lethal tumor that is highly resistent to therapy, and achieving a cure through surgical intervention is difficult. Previous research has shown that therapeutic resistance of GBM is caused by the selective survival of highly tumorigenic GSCs (9). It is critical for cancer therapy that treatments target and eliminate these resistent population of stem-like cells (29). Therefore, it is necessary to culture and enrich GSCs in GBM cells in order to identify their aggressive mechanisms and then aim to eliminate GSCs in patients.

In the present study, we successfully isolated GBMS from the human GBM cell line WJ1. GBMS exhibited properties of GSCs, including the ability for self-renewal, exhibited pale with toluidine blue, expression of known GSC markers and high tumorigenicity in vivo. These lines of evidence confirmed the isolation of GSCs from WJ1.

A previous study showed that GSCs play core roles in the therapeutic resistance of GBM (9). Thus, targeting malig- 
nant GSCs using therapeutic drugs that have the potential to eliminate the most aggressive GSCs but preserve neural cell populations should be the preferred treatment measure in experimental research. Tanshinone IIA has anticancer $(30,31)$ and anti-inflammatory $(14,17,18)$ activities. In the present study, following GSC exposure to tanshinone IIA, the formation of neurospheres was reduced in a dose-dependent manner and the cell viability of GSCs was suppressed in a dose- and timedependent manner. These results suggest that tanshinone IIA has strong anti-GSC activity. In an in vivo experiment, tanshinone IIA slowed the growth of human brain cancer derived from GSCs, and significantly reduced the weight and volume of tumor masses without any untoward toxicity. These findings demonstrated that using tanshinone IIA has a potential inhibitory effect on GSCs in vitro and in vivo.

Since CSCs play a core role in cancer recurrence, metastasis and high patient mortality, targeting and attenuating CSC stemness, induction of CSC differentiation and apoptosis are promising treatment avenues other than targeting tumor masses in the treatment of a malignant tumors. Friedman et al (32) demonstrated that all-trans retinoic acid (ATRA), a derivative of retinol, causes differentiation of CSCs as well as normal neural progenitor cells by the loss of the stem cell marker nestin. In the present study, expression levels of stemness markers (CD133 and nestin) in GSCs were decreased, and differentiation and neural lineage markers (GFAP and $\beta$ IIItubulin) were increased in vitro and in vivo in a dose-dependent manner after treatment with tanshinone IIA. This indicates that the stemness of GSCs was attenuated after treatment with tanshinone IIA. The Annexin V analysis and western blot analysis of apoptotic proteins Bax and cleaved caspase-3 and anti-apoptotic protein Bcl-2 indicated that tanshinone IIA treatment resulted in apoptotic cell death of GSCs. These results suggest that tanshinone IIA has an anti-GSC activity through induction of GSC apoptosis. Taken together, all these data strongly demonstrated that tanshinone IIA has the potential not only to attenuate GSC stemness, but also to induce GSC apoptosis.

It was well recognized that inflammatory cytokines and signaling pathways play pivotal roles in maintaining stemlike properties in human glioma cells (33). Wang et al (34) demonstrated that GSCs preferentially express the IL6 receptors $\alpha$ (IL6R $\alpha$ ) and glycoprotein 130 (gp130), and the targeting of IL6R $\alpha$ or IL6 ligand expression in GSCs significantly reduces growth and neurosphere formation capacity while increasing apoptosis. Guryanova et al (35) demonstrated that the activation of STAT3 signaling is necessary for maintaining self-renewal and the tumorigenic potential of GSCs. STAT3 is a downstream mediator of pro-survival IL6 signals in GSCs and is constitutively activated in glioma cells. Perturbation of IL6 signaling in GSCs attenuates STAT3 activation, and small molecule inhibitors of STAT3 potently induce GSC apoptosis (34). Thus, it can be seen that the IL6/STAT3 signaling axis plays an important role for GSC maintanence and activity. In the present study, the expression level of IL6 was higher in GSCs and tumor tissue derived from GSC bearing-mice. IL6 levels were downregulated after treatment with tanshinone IIA, indicating that tanshinone IIA has a significant inhibitory effect on IL6 expression in GSCs. The oncogenic potential of STAT3 depends on its phosphorylation on tyrosine705 and serine727 that leads to activation of target gene transcription. Phosphorylated STATs form dimers, translocate to the nucleus using the karyopherin- $\beta$ nucleocytoplasmic transport system, bind DNA, and activate transcription $(36,37)$. In the present study, activated STAT3 [(STAT3(tyrosine705) and STAT3(serine727)] had high levels in GSCs. Tanshinone IIA not only downregulated the expression levels of IL6 but also decreased activated STAT3 in GSCs in vitro and in vivo in a dose-dependent manner. This result suggests that the IL6/STAT3 signaling axis might mediate the growth and progression of GSCs. Disturbing IL6/STAT3 signaling pathways by tanshinone IIA is closely associated with growth inhibition of GSCs,

Based on the findings in this experimental study, tanshinone IIA inhibits proliferation, attenuates stemness and induces apoptosis in GSCs in vitro and in vivo. Its partial mechanism of activity may be associated with attenuating inflammatory cytokine production and blocking the IL6/STAT3 signaling pathway.

\section{Acknowledgements}

This research was supported by the National Natural Science Foundation of China (grant no. 31071197).

\section{References}

1. Daumas-Duport C, Scheithauer B, O'Fallon J and Kelly P: Grading of astrocytomas, a simple and reproducible method. Cancer 62: 2152-2165, 1988.

2. Smith JS and Jenkins RB: Genetic alterations in adult diffuse glioma: occurrence, significance, and prognostic implications. Front Biosci 5: D213-D231, 2000.

3. Wang L, Liu Z, Balivada S, Shrestha T, Bossmann S, Pyle M, Pappan L, Shi J and Troyer D: Interleukin-1 $\beta$ and transforming growth factor- $\beta$ cooperate to induce neurosphere formation and increase tumorigenicity of adherent LN-229 glioma cells. Stem Cell Res Ther 3: 5, 2012.

4. Jordan CT: Cancer stem cells: controversial or just misunderstood? Cell Stem Cell 4: 203-205, 2009.

5. Ignatova TN, Kukekov VG, Laywell ED, Suslov ON, Vrionis FD and Steindler DA: Human cortical glial tumors contain neural stem-like cells expressing astroglial and neuronal markers in vitro. Glia 39: 193-206, 2002.

6. Singh SK, Hawkins C, Clarke ID, Squire JA, Bayani J, Hide T, Henkelman RM, Cusimano MD and Dirks PB: Identification of human brain tumour initiating cells. Nature 432: 396-401, 2004.

7. Galli R, Binda E, Orfanelli U, Cipelletti B, Gritti A, De Vitis S, Fiocco R, Foroni C, Dimeco F and Vescovi A: Isolation and characterization of tumorigenic, stem-like neural precursors from human glioblastoma. Cancer Res 64: 7011-7021, 2004.

8. Bao S, Wu Q, McLendon RE, Hao Y, Shi Q, Hjelmeland AB, Dewhirst MW, Bigner DD and Rich JN: Glioma stem cells promote radioresistance by preferential activation of the DNA damage response. Nature 444: 756-760, 2006.

9. Bao S, Wu Q, Sathornsumetee S, Hao Y, Li Z, Hjelmeland AB, Shi Q, McLendon RE, Bigner DD and Rich JN: Stem cell-like glioma cells promote tumor angiogenesis through vascular endothelial growth factor. Cancer Res 66: 7843-7848, 2006.

10. Eramo A, Ricci-Vitiani L, Zeuner A, Pallini R, Lotti F, Sette G, Pilozzi E, Larocca LM, Peschle C and De Maria R: Chemotherapy resistance of glioblastoma stem cells. Cell Death Differ 13: 1238-1241, 2006.

11. Rich JN: Cancer stem cells in radiation resistance. Cancer Res 67: 8980-8984, 2007.

12. Liu G, Yuan X, Zeng Z, Tunici P, Ng H, Abdulkadir IR, Lu L, Irvin D, Black KL and Yu JS: Analysis of gene expression and chemoresistance of $\mathrm{CD} 133^{+}$cancer stem cells in glioblastoma. Mol Cancer 5: 67, 2006.

13. Singh SK, Clarke ID, Hide T and Dirks PB: Cancer stem cells in nervous system tumors. Oncogene 23: 7267-7273, 2004. 
14. Yin X, Yin Y, Cao FL, Chen YF, Peng Y, Hou WG, Sun SK and Luo ZJ: Tanshinone IIA attenuates the inflammatory response and apoptosis after traumatic injury of the spinal cord in adult rats. PLoS One 7: e38381, 2012.

15. Dai ZK, Qin JK, Huang JE, Luo Y, Xu Q and Zhao HL: Tanshinone IIA activates calcium-dependent apoptosis signaling pathway in human hepatoma cells. J Nat Med 66: 192-201, 2012.

16. Chiu TL and Su CC: Tanshinone IIA induces apoptosis in human lung cancer A549 cells through the induction of reactive oxygen species and decreasing the mitochondrial membrane potential. Int J Mol Med 25: 231-236, 2010.

17. Ren ZH, Tong YH, Xu W, Ma J and Chen Y: Tanshinone IIA attenuates inflammatory responses of rats with myocardia infarction by reducing MCP-1 expression. Phytomedicine 17: 212-218, 2010

18. Fan GW, Gao XM, Wang H, Zhu Y, Zhang J, Hu LM, Su YF, Kang LY and Zhang BL: The anti-inflammatory activities of tanshinone IIA, an active component of TCM, are mediated by estrogen receptor activation and inhibition of iNOS. J Steroid Biochem Mol Biol 113: 275-280, 2009.

19. Chen W, Tang F, Xie B, Chen S, Huang H and Liu P: Amelioration of atherosclerosis by tanshinone IIA in hyperlipidemic rabbits through attenuation of oxidative stress. Eur J Pharmacol 674: 359-364, 2012.

20. Wang X, Wei Y, Yuan S, Liu G, Lu Y, Zhang J and Wang W: Potential anticancer activity of tanshinone IIA against human breast cancer. Int J Cancer 116: 799-807, 2005.

21. Su CC and Lin YH: Tanshinone IIA inhibits human breast cancer cells through increased Bax to Bcl-xL ratios. Int J Mol Med 22: 357-361, 2008.

22. Iliopoulos D, Hirsch HA, Wang G and Struhl K: Inducible formation of breast cancer stem cells and their dynamic equilibrium with non-stem cancer cells via IL6 secretion. Proc Natl Acad Sci USA 108: 1397-1402, 2011.

23. Korkaya H, Liu S and Wicha MS: Regulation of cancer stem cells by cytokine networks: attacking cancer's inflammatory roots. Clin Cancer Res 17: 6125-6129, 2011.

24. Jang SI, Jeong SI, Kim KJ, Kim HJ, Yu HH, Park R, Kim HM and You YO: Tanshinone IIA from Salvia miltiorrhiza inhibits inducible nitric oxide synthase expression and production of TNF-alpha, IL-1beta and IL6 in activated RAW 264.7 cells. Planta Med 69: 1057-1059, 2003.

25. Wang J, Wang X, Jiang S, Lin P, Zhang J, Wu Y, Xiong Z, Ren JJ and Yang H: Establishment of a new human glioblastoma multiforme cell line (WJ1) and its partial characterization. Cell Mol Neurobiol 27: 831-843, 2007.

26. Lee J, Kotliarova S, Kotliarov Y, Li A, Su Q, Donin NM Pastorino S, Purow BW, Christopher N, Zhang W, Park JK and Fine HA: Tumor stem cells derived from glioblastomas cultured in bFGF and EGF more closely mirror the phenotype and genotype of primary tumors than do serum-cultured cell lines. Cancer Cell 9: 391-403, 2006.
27. van Meerloo J, Kaspers GJ and Cloos J: Cell sensitivity assays: the MTT assay. Methods Mol Biol 731: 237-245, 2011.

28. Kim MJ, Kim YJ, Park HJ, Chung JH, Leem KH and Kim HK: Apoptotic effect of red wine polyphenols on human colon cancer SNU-C4 cells. Food Chem Toxicol 44: 898-902, 2006.

29. Yuan X, Curtin J, Xiong Y, Liu G, Waschsmann-Hogiu S, Farkas DL, Black KL and Yu JS: Isolation of cancer stem cells from adult glioblastoma multiforme. Oncogene 23: 9392-9400, 2004.

30. Kapoor S: Tanshinone IIA: a potent, natural anti-carcinogenic agent for the management of systemic malignancies. Chin J Integr Med 15: 153, 2009.

31. Ma H, Fan Q, Yu J, Xin J and Zhang C: Novel microemulsion of tanshinone IIA, isolated from Salvia miltiorrhiza Bunge, exerts anticancer activity through inducing apoptosis in hepatoma cells. Am J Chin Med 41: 197-210, 2013.

32. Friedman MD, Jeevan DS, Tobias M, Murali $R$ and Jhanwar-Uniyal M: Targeting cancer stem cells in glioblastoma multiforme using mTOR inhibitors and the differentiating agent all-trans retinoic acid. Oncol Rep: 30: 1645-1850, 2013.

33. Jin X, Kim SH, Jeon HM, Beck S, Sohn YW, Yin J, Kim JK, Lim YC, Lee JH, Kim SH, Kang SH, Pian X, Song MS, Park JB, Chae YS, Chung YG, Lee SH, Choi YJ, Nam DH, Choi YK and $\mathrm{Kim} \mathrm{H}$ : Interferon regulatory factor 7 regulates glioma stem cells via interleukin-6 and Notch signalling. Brain 135: 1055-1069, 2012.

34. Wang H, Lathia JD, Wu Q, Wang J, Li Z, Heddleston JM, Eyler CE, Elderbroom J, Gallagher J, Schuschu J, MacSwords J, Cao Y, McLendon RE, Wang XF, Hjelmeland AB and Rich JN: Targeting interleukin 6 signaling suppresses glioma stem cell survival and tumor growth. Stem Cells 27: 2393-2404, 2009.

35. Guryanova OA, Wu Q, Cheng L, Lathia JD, Huang Z, Yang J, MacSwords J, Eyler CE, McLendon RE, Heddleston JM, Shou W, Hambardzumyan D, Lee J, Hjelmeland AB, Sloan AE, Bredel M, Stark GR, Rich JN and Bao S: Nonreceptor tyrosine kinase BMX maintains self-renewal and tumorigenic potential of glioblastoma stem cells by activating STAT3. Cancer Cell 19: 498-511, 2011.

36. Bromberg J and Darnell JE Jr: The role of STATs in transcriptional control and their impact on cellular function. Oncogene 19: 2468-2473, 2000.

37. Schindler C, Levy DE and Decker T: JAK-STAT signaling: from interferons to cytokines. J Biol Chem 282: 20059-20063, 2007. 